\title{
Deslocalización de espacios y tiempos formativos universitarios con
}

\section{TIC}

\section{Delocalisation of University training spaces and times with ICT}

\author{
María Luisa Sevillano-García*, Esteban Vázquez-Cano*, María Ángeles Pascual Sevillano** \\ Universidad Nacional de Educación a Distancia*, Universidad de Oviedo**
}

\begin{abstract}
Resumen
El Informe Horizonte 2020 (H2020) estimula la innovación como objetivo, indicando aprovechar potencial y posibilidades de las modernas tecnologías. Objetivo de esta investigación fue analizar lugares y usos en los que estudiantes universitarios españoles e hispanoamericanos utilizan dispositivos digitales móviles con fines educativos. Concluimos que su aprovechamiento en el mundo hispano se concentra en los smartphones Las diferencias más significativas indican que en España se utiliza más el smartphone en las aulas y el ordenador portátil fuera del recinto universitario. En Hispanoamérica registra mayor frecuencia el smartphone fuera del recinto universitario y el ordenador portátil dentro para fines educativos.

Palabras Clave. Deslocalización, ubicuidad, movilidad, espacios, nuevos estilos
\end{abstract}

\begin{abstract}
The Horizon 2020 Report (H2020) fosters innovation as an objective, showing the potential and possibilities of modern technologies. The objective of this research was to analyze in which places and educational uses of mobile digital devices Spanish and Latinamerican university students do. We conclude that the main digital device in the Hispanic world was the smartphone. The most significant differences indicate that in Spain the smartphone is used frequently in classrooms and the laptop outside the campus. In Latinamerica the smartphone is used more frequently outside the campus and the laptop inside the campus for educational purposes. Key words: Delocalisation, Ubiquity, mobility, spaces, new educational styles.
\end{abstract}

\section{Una situación nueva en el aprendizaje universitario}

El aprendizaje móvil es un campo innovador de investigación y práctica educativa en acuciante urgencia. Sin embargo, existe todavía poco trabajo empírico , teórico y conceptual con el que explicar la compleja relación entre las características de la evolución tecnológica rápida y, a veces revolucionaria, su potencial de educación y aprendizaje, así como su integración en la vida cotidiana de los usuarios. En el Informe Horizonte 2020(H2020) ( 2013 ) Programa Marco de Investigación e Innovación de la Unión Europea para el periodo 2014-2020, se estimula a cultivar en la enseñanza aprendizaje la innovación como objetivo. indicando aprovechar el potencial innovador creativo y productivo de todas las generaciones y las posibilidades que ofrecen las modernas tecnologías. De esta forma se puede ayudar en la práctica profesional a profesores y formadores.

Los procesos de enseñanza-aprendizaje y la interacción social entre los estudiantes en la Educación Superior ya no sólo se realiza en espacios físicos y determinados por los campus universitarios El espacio y el lugar en el que el estudiante se encuentra no es un elemento determinante para poder realizar intercambios académicos o personales que pueden formar parte del proceso de enseñanza-aprendizaje en el contexto universitario actual; un contexto caracterizado por los principios de movilidad y ubicuidad que favorecen las infraestructuras tecnológicas para la conexión de dispositivos digitales (Gegenfurtner, Veermans, \& Vauras, 2013; Mercier \& Higgins, 2013; Sevillano \& Vázquez-Cano, 2015).

Los análisis que se han realizado sobre la ubicuidad y el uso de los dispositivos digitales móviles en diferentes partes del mundo se han centrado principalmente en el uso educativo y la potencialidad didáctica de estos dispositivos Asimismo, los informes que diferentes compañías tecnológicas han realizado se basan en el análisis de patrones de uso de los dispositivos conforme a diferentes variables: edad y sexo de los usuarios, número y carácter de las aplicaciones instaladas y utilizadas, frecuencia y franjas horarias de conexión, tiempos de conexión, etc. (UNESCO, 2013; Nielsen, 2013; ADOBE, 2014; Deloitte, 2014; Ericsson, 2014ab; Webcertain, 2014; GSMA, 2014. Por el contrario, el análisis de espacios y lugares desde donde el usuario hace uso de los dispositivos digitales móviles, se ha realizado desde una perspectiva más restrictiva y casi limitada al aspecto geográfico-urbano con aplicaciones de geolocalización (Kamarainen et al., 2012; Liao, 2015). La influencia de los espacios y lugares desde el que el usuario se conecta a un dispositivo móvil y el fin con el que lo hace no ha sido apenas estudiado en el ámbito educativo ( Dennen \& Hao, 2014). 


\section{Diseño científico del estudio}

El objetivo de la investigación que realizamos y de cuyo informe general, esta aportación es una parte, fue identificar y analizar los espacios y lugares en los que una muestra significativa de estudiantes universitarios españoles e hispanoamericanos utilizan sus dispositivos digitales móviles (smartphones, y ordenadores portátiles) con fines educativos Nos servimos de de una muestra intercontinental de 805 estudiantes universitarios distribuidos de la forma siguiente:

\begin{tabular}{l|l}
\hline \multicolumn{2}{l}{ Cuestionarios recibidos y tratados } \\
\hline Universidades & Protocolos \\
\hline Complutense & 21 \\
\hline Vigo & 46 \\
\hline Oviedo & 169 \\
\hline Granada & 77 \\
\hline UNED & 108 \\
\hline $\begin{array}{l}\text { Chile. Universidad del Libertador } \\
\text { Bernardo O’higgins.. Santiago de } \\
\text { Chile }\end{array}$ & 98 \\
\hline $\begin{array}{l}\text { Perú. Universidad Nacional } \\
\text { Hermilio Valdizán. Huánuco. }\end{array}$ & 42 \\
\hline $\begin{array}{l}\text { Colombia. Universidad de } \\
\text { Cartagena }\end{array}$ & 110 \\
\hline $\begin{array}{l}\text { Panamá. Universidad Pública de } \\
\text { Panamá }\end{array}$ & 29 \\
\hline $\begin{array}{l}\text { México. Universidad } \\
\text { Veracruzana. Xalapa }\end{array}$ & 105 \\
\hline TOTAL & $\mathbf{8 0 5}$ \\
\hline $\begin{array}{l}\text { 421 pertenecen a la muestra de España y 384 a } \\
\text { Hispanoamérica.. }\end{array}$ \\
\hline
\end{tabular}

\section{Instrumento}

Elaborado y validado por el sistema de expertos un cuestionario con preguntas cerradas y abiertas fue aplicado por los profesores correspondientes en las diversas Universidades Para esta aportación hemos seleccionado dos preguntas relacionadas y pertinentes con los objetivos pretendidos Este instrumento ha sido registrado oficialmente en el Ministerio de Economía y Competitividad de España, habiendo sido traducido al alemán e Inglés y aplicado en diversos países entre ellos China. Título de Registro de Marca. Fecha de concesión 24 de julio de 2014.MARCA.N ${ }^{\circ} 3.502 .443$ DISTINTIVO MAUDIMO CLASE 41. EDUCACIÓN, FORMACIÓN Y ACTIVIDADES CULTURALES Cronbach's Alpha for the overall scale: 0.920 Pregunta - Selecciona los lugares desde los cuales utilizas el ordenador portátil y/o el teléfono móvil y la frecuencia de uso según la escala tipo likert ( $1=$ ninguna frecuencia, 2= alguna vez al mes, $3=$ alguna vez a la semana, $4=$ todos los días) y las utilidades que obtiene.
Resultados e interpretación Lugares preferidos

\begin{tabular}{|c|c|c|c|c|}
\hline & \multicolumn{2}{|c|}{$\begin{array}{lll}\text { Cafetería de la } \\
\text { Facultad }\end{array}$} & Pasillos & \multirow[b]{2}{*}{$\begin{array}{l}\text { Tel. } \\
\text { móvil }\end{array}$} \\
\hline & $\begin{array}{l}\text { Ord. } \\
\text { Portátil }\end{array}$ & $\begin{array}{l}\text { Tel. } \\
\text { móvil }\end{array}$ & $\begin{array}{l}\text { Ord. } \\
\text { Portátil }\end{array}$ & \\
\hline España & 5.0 & 20.7 & 23.2 & 6.1 \\
\hline Chile & 2.8 & 24.4 & 38.6 & 1.5 \\
\hline Perú & 6.7 & 22.9 & 38.2 & 2.2 \\
\hline Colombia & 22.7 & 31.2 & 40.4 & 13.7 \\
\hline Panamá & 7.1 & 31.0 & 35.7 & 3.6 \\
\hline México & 6.1 & 23.7 & 31.3 & 12.2 \\
\hline \multicolumn{2}{|c|}{ Aulas } & \multicolumn{3}{|c|}{ Biblioteca } \\
\hline & $\begin{array}{l}\text { Ord. } \\
\text { Portátil }\end{array}$ & $\begin{array}{l}\text { Tel. } \\
\text { móvil }\end{array}$ & $\begin{array}{l}\text { Ord. } \\
\text { Portátil }\end{array}$ & $\begin{array}{l}\text { Tel. } \\
\text { móvil }\end{array}$ \\
\hline paña & & 20.1 & 18.3 & 16.4 \\
\hline iile & & 25.0 & 20.6 & 13.1 \\
\hline rú & & 30.8 & 13.3 & 17.1 \\
\hline lombia & & - & 19.0 & 34.6 \\
\hline namá & & 42.9 & 14.6 & 6.3 \\
\hline \multirow[t]{3}{*}{ México } & & 25.3 & 16.7 & 9.2 \\
\hline & \multicolumn{2}{|c|}{ Lugares de ocio } & \multicolumn{2}{|l|}{ En casa } \\
\hline & $\begin{array}{l}\text { Ord. } \\
\text { Portátil }\end{array}$ & $\begin{array}{l}\text { Tel. } \\
\text { móvil }\end{array}$ & $\begin{array}{l}\text { Ord. } \\
\text { Portátil }\end{array}$ & $\begin{array}{l}\text { Tel. } \\
\text { móvil }\end{array}$ \\
\hline España & 6.3 & 26.1 & 23.7 & 22.9 \\
\hline Chile & 5.6 & 47.6 & 6.9 & 11.9 \\
\hline Perú & 10.7 & 32.4 & 30.0 & 47.1 \\
\hline Colombia & 21.8 & 29.9 & 30.8 & 34.8 \\
\hline Panamá & 9.1 & 32.0 & 19.2 & 38.5 \\
\hline México & 14.0 & 31.6 & 29.4 & 28.6 \\
\hline \multicolumn{5}{|c|}{ En el medio de transporte / autobús, metro, tren } \\
\hline & \multicolumn{2}{|c|}{ Ord. Portátil } & \multicolumn{2}{|c|}{ Tel. móvil } \\
\hline España & \multicolumn{2}{|l|}{$2 . .7$} & \multicolumn{2}{|l|}{27.6} \\
\hline Chile & \multicolumn{2}{|l|}{1.0} & \multicolumn{2}{|l|}{14.3} \\
\hline Perú & \multicolumn{2}{|l|}{3.7} & \multicolumn{2}{|l|}{40.0} \\
\hline Colombia & \multicolumn{2}{|l|}{1.2} & \multicolumn{2}{|l|}{10.0} \\
\hline Panamá & \multicolumn{2}{|l|}{1.1} & \multicolumn{2}{|l|}{22.7} \\
\hline México & \multicolumn{2}{|l|}{0.4} & \multicolumn{2}{|l|}{28.6} \\
\hline
\end{tabular}

Estudiamos en este apartado los espacios preferidos, Es importante resaltar que los dispositivos empleados permiten, sin necesidad de conexión la movilidad. La cafetería no es lugar de referencia con el ordenador portátil, salvo en el caso de Colombia , con un $22.7 \%$ para el portátil y $31.2 \%$ con el teléfono móvil. Este dispositivo si experimenta mayor utilización. En cambio los pasillos de la Facultad gozan de preferencia a la hora de emplear el ordenador portátil. Los autores conocen la Universidad donde se aplicó el cuestionario y saben que realmente dispone de pasillos amplios y bien dotados de mesas y sillas. El uso en las aulas es alto tanto para el ordenador como para el teléfono. La biblioteca tampoco resulta ser el espacio de gran preferencia , salvo en el caso de Colombia con el 53.6 $\%$ para ambos instrumentos.

Los tiempos pasados en espacios extraacadémicos como lugares de ocio, la casa o el medio de transporte son incorporados mediante los dispositivos móviles a la ampliación del tiempo y oportunidad de estudio. 
Globalmente el teléfono móvil es el preferido en Perú $40.0 \%$ destacan por aprovechar el tiempo que pasan en los transportes públicos con el teléfono móvil. Sin embargo hay para quienes el Smartphone es el instrumento ideal. En los lugares de ocio vuelve a ser el teléfono móvil el instrumento preferido, destacando Chile con un $47.6 \%$.

\section{Utilidades académicas}

El ordenador portátil ocupa puestos más altos que el teléfono móvil en su empleo para elaborar trabajos académicos En horario escolar utilizo el ordenador portátil para realizar las prácticas y ejercicios de las asignaturas ( protocolo 341 ). El teléfono móvil, salvo Perú y Colombia en la categoría de a menudo, es escasa. Panamá destaca sobre todos con un $82.1 \%$. en la categoría de siempre, categoría en la que todos los países muestran un alto grado de uso .Respecto al teléfono móvil, los valores en la categoría de siempre son menores que en el caso del portátil. España registra el más bajo y tiene relación con la dificultad de conexión debido al alto coste económico, $0.4 \%$. Colombia, y Panamá aparecen como los países en los que los estudiantes utilizan más a menudo para estudiar, el teléfono móvil, pero siempre con valores menores a los registrados en relación con el ordenador portátil, dispositivo empleado para el estudio a menudo con altos valores por todos los encuestados, destacando el caso de México con el $38.2 \%$. Para llevar a cabo mi estudio empleo los apuntes y temario dado en clase y recogido en el ordenador portátil y a veces en el móvil, que suelo completar con información obtenida de Internet para resolver mis dudas, ( protocolo 62). El ordenador portátil lo utilizo en mi casa, para buscar la información que pueda necesitar para realizar los trabajos para mis clases, para pasar a limpio los apuntes que hasta principios de este año escribía a bolígrafo . ( Protocolo 199 ).

La información académica que implica, becas, calificaciones, exámenes, apuntes, recibe frecuentes usos con el ordenador portátil por parte de todos los grupos encuestados. A la hora de hacer trabajos, muchas veces he utilizado el móvil tanto para recopilar información de forma instantánea dada su velocidad e incluso para ayudarme de sus aplicaciones para hacerlos ( Protocolo 92 ). España con el 34.3\% sobresale de los demás. Colombia y Perú con $33.8 \%$ y el $25.7 \%$ son los países donde más se aprovechan los estudiantes para recabar este tipo de información. Chile con el 52.4\% y Colombia con el $40.6 \%$ representan los países en los que la categoría de siempre es mayoritaria en el uso del ordenador portátil En el uso del teléfono móvil se registran valores de nada o casi nada muy altos en todos los países. $47.3 \%$ Perú y 35.5 España \%. El ordenador portátil a la hora de intercambiar apuntes, es más frecuente en Chile y España El teléfono móvil ocupa lugares significativos en Panamá 27.3\%, superando al portátil ( $8.3 \%$ ) y en Perú $23.7 \%$, El teléfono móvil, le utilizo para ver y enviar apuntes y para cualquier urgencia que tenga que ver con lo académico. De hecho le tengo siempre conectado y aunque le ponga en silencio estoy siempre a disposición ( protocolo 100 ) Los valores más altos en la categoría de siempre con respecto al ordenador portátil los encontramos en Chile, $42.7 \%$ y en Colombia $39.2 \%$ En las categorías de nada y casi nada a la hora de intercambiar apuntes vía teléfono móvil sobresale España con registros de $83.3 \%$. Tal vez la frecuente insistencia en la competitividad lleva a no intercambiar el producto académico.

Las categorías de nada o casi nada son altas en todos los países destacando Perú que suma en ambas $45.9 \%$ y España $43.5 \%$. Utilizo el teléfono móvil para concertar citas con mis compañeros de facultad a la hora de hacer trabajos, así como para el intercambio de información de cualquier tipo ( Protocolo 80 ) El ordenador portátil, nos da independencia para poder movernos de un lado a otro y nos permite coger apuntes en la universidad, hacer trabajos de grupo sin necesidad de estar en un recinto cerrado. Lo utilizo para leer la prensa, completar apuntes, estar informada, conseguir apuntes colgados en la página Web de la universidad ( protocolo 3 )

La rotación oblicua de los factores para su interpretación. Ese cambio de ejes nos ayuda a separar y discriminar mejor cómo se relacionan con ellos las variables. En tal situación el programa SPSS muestra la salida de dos matrices, a diferencia del caso de la rotación ortogonal donde sólo muestra la matriz de factores rotados. En nuestro caso, la salida informa de la Matriz de Configuración que recoge los pesos directos de cada factor sobre las variables (es decir, los pesos del modelo factorial oblicuo). Y además, también informa de la Matriz de Estructura que muestra las correlaciones de cada variable con el factor oblicuo recogiendo los efectos directos del factor sobre la variable, como los indirectos de otros factores a través de la correlación con un factor dado. Para la interpretación de la rotación oblicua ha de tenerse en cuenta ambas matrices, de manera que la construcción de significación de los factores es algo más compleja que para la rotación factorial.

El Factor 1 (Uso educativo del smartphone en el recinto universitario) presenta diferencias significativas entre las dos zonas geográficas. En España se utiliza más el smartphone en las aulas. El porcentaje acumulado de los estudiantes españoles que lo hacen "a menudo" o "siempre" es del $42 \%$, en comparación con otros países hispanoamericanos Colombia (17,8\%), Panamá (21,4\%), Perú (22\%), México $(16,75)$ y Chile $(8,6 \%)$. El Factor 2 (Uso educativo del smartphone fuera del recinto universitario), presenta un mayor uso educativo en los estudiantes hispanoamericanos que los españoles. La media de uso en los países hispanoamericanos analizados hace un uso educativo de este dispositivo más intenso ("a menudo" y "siempre") (26,03\%) que los españoles (16,65\%). El Factor 3 (Uso educativo del ordenador portátil fuera del recinto universitario) muestra diferencias significativas entre los estudiantes de las dos zonas geográficas. Los estudiantes españoles emplean el ordenador portátil fuera del recinto educativo con mayor frecuencia que los estudiantes hispanoamericanos $(+13,6 \%)$. El Factor 4 (Uso 
educativo del portátil en el recinto universitario) muestra que los estudiantes hispanoamericanos utilizan más sus ordenadores portátiles para fines educativos dentro del recinto universitario que los españoles $(+5,16 \%)$.

\section{Conclusiones}

Los resultados del análisis estadístico factorial muestran que el uso educativo de los dispositivos digitales móviles en el mundo hispano se concentra en los smartphones. En este caso, los lugares más frecuentes son los pasillos de facultad, las aulas y la biblioteca. Con menor significación estadística se hace un uso educativo del smartphone fuera del recinto universitario principalmente desde los medios de transporte, las zonas de ocio al aire libre, el domicilio habitual, el lugar de trabajo y la calle. Los estudiantes también utilizan fuera del recinto escolar con fines educativos el ordenador portátil, desde el domicilio habitual y las zonas de ocio al aire libre y, el segundo, desde una localización más variada: domicilio habitual, medios de transporte, lugar de trabajo, zonas de ocio al aire libre y en la calle. Los resultados de las pruebas paramétricas y no paramétricas para la comparación inter-grupos en las dos zonas geográficas nos han permitido determinar si existen diferencias de uso en los diferentes países. El estadístico de contraste "Mann-Whitney" muestra diferencias significativas entre españoles e hispanoamericanos en tres factores: Factor 1. Uso educativo del smartphone en el recinto universitario, Factor 2. Uso educativo del smartphone fuera del recinto universitario y Factor 3. Uso educativo del portátil fuera del recinto universitario. Asimismo, la prueba t-student muestra diferencias significativas para el Factor 4. Uso educativo del portátil en el recinto universitario. Las diferencias más significativas mostradas en la tabla de contingencia nos permiten observar que en España se utiliza más con fines educativos el smartphone en las aulas y el ordenador portátil fuera del recinto universitario. Por el contrario, en Hispanoamérica se emplea con mayor frecuencia el smartphone fuera del recinto universitario y el ordenador portátil dentro del recinto universitario para fines educativos.

Estos resultados instan a las instituciones, profesores y responsables educativos a una mejora constante de los procesos didácticos, de los contenidos y de los formatos en los que se ofrecen estos contenidos así como las formas de interacción y trabajo colaborativo. El conocimiento del patrón de conexión conforme al espacio y lugar desde el que se conecta el dispositivo digital móvil de un estudiante con fines educativos puede aprovecharse para el diseño de actividades sensibles al contexto que enriquezcan la experiencia formativa y contextualicen el contenido teórico con recursos como la realidad aumentada o los grupos colaborativos en red. Las posibilidades son numerosas y diversas conforme al carácter de los estudios y las asignaturas implicadas, aunque las dificultades son también importantes, entre las que destacan, la privacidad de las comunicaciones, la formación del profesorado, la inversión en infraestructuras tecnológicas y la actualización hacia diseños tecnodidácticos avanzados que entronquen con la realidad social y profesional de la sociedad actual. Los resultados presentados representan a nuestro entender una triple urgencia y demanda. La primera afectaría a las entidades propietarias de las telecomunicaciones para que en aras de ir aminorando la brecha digital y favorecer los usos académicos, instruccionales, de las modernas tecnologías móviles y ubicuas ofrezcan tarifas más reducidas a los estudiantes, como segmento débil en capacidad adquisitiva, principalmente en aquellos países en los que se han convertido en las casi únicas herramientas para acceder al conocimiento. Se ha dado el salto al universo digital, pero su acceso sigue siendo limitado por los altos costes de conexión, uso y mantenimiento. En segundo término y ligado al mismo sector estaría en las baterías. Se precisa investigación y experimentación para que éstas vayan teniendo más autonomía. La segunda instancia estaría en el profesorado universitario. Los usos frecuentes y crecientes por parte de los estudiantes piden más contenidos curriculares en las redes y no solo y especialmente informaciones aunque sean académicas. $\mathrm{Si}$ bien el intercambio de apuntes o trabajos se acredita como frecuente y útil, es menester propiciar una mayor información docente sobre los recursos digitalizados ya existentes en todas las áreas de conocimiento, para que los estudiantes desde sus móviles accedan a los mismos. En esta situación digital no podemos tener esos inmensos repositorios de recursos fosilizado por falta de conocimiento, tiempo o carencia de preparación o interés profesoral. El propio consorcio Erasmus está diseñando un gran portal multilingüe entre las más de 4000 instituciones que lo integran para la difusión de cursos masivos con el objetivo de interconectar conocimiento, investigación y transferencia de resultados entre las universidades y donde el formato audiovisual ubicuo y móvil será uno de los prioritarios ( Vázquez Cano, E. 2013: 90 ) Entendemos que es menester diseñar un sistema de análisis tanto para la producción, como la explotación de los recursos a los que se puede acceder a través de los dispositivos móviles en la vida académica de estudiantes, profesores e instituciones superiores de estudio como son las Universidades. Esto nuevos medios posibilitan y urgen una nueva forma de organizar, representar y codificar la realidad. En consecuencia es necesario desarrollar elementos de análisis crítico y de una formación de los estudiantes que les permita utilizar esa información de manera adecuada ( Sevillano García, 2014: 297 )

\section{Fuentes}

ADOBE (2014). U.S. Mobile Benchmark Report
ADOBE
http://www.cmo.com/content/dam/CMO_Other/ADI/
$\begin{gathered}\text { ADI_Mobile_Report_2014/2014_US_Mobile_Bench } \\ \text { mark_Report.pdf }\end{gathered}$
$\begin{gathered}\text { Deloitte (2014). Consumo Móvil en España 2014 } \\ \text { evolución. }\end{gathered}$
Revolución
y


http://www2.deloitte.com/es/es/pages/technologymedia-and-telecommunications/articles/consumomedios-espana.html

Dennen, V. P., \& Hao, S. (2014). Intentionally mobile pedagogy: the M-COPE framework for mobile learning in higher education. Technology, Pedagogy and Education, 23(3), 397-419.

Ericsoon (2014a). EUROPE ERICSSON MOBILITY REPORT APPENDIX. http://www.ericsson.com/res/docs/2014/emrjune2014-regional-appendices-europe.pdf

Ericsson (2014b). Ericsson Mobility Report ON THE PULSE OF THE NETWORKED SOCIETY.http://www.ericsson.com/mobility-report

Gegenfurtner, A., Veermans, K., \& Vauras, M. (2013). Effects of computer support, collaboration, and time lag on performance self-efficacy and transfer of training: A longitudinal meta-analysis. Educational Research Review, 8, 75-89.

GSMA (2014) The Mobile Economy 2014. http://www.gsmamobileeconomy.com/

Kamarainen, A.; Metcalf, S.; Grotzer, T.; Browne, A.; Mazzuca, D.; Tutweiler, M.S. y Dede, C. (2012). Ecomobile: Integrating augmented reality and probe ware with environmental education field trips. Computers \& Education, 68, 545-556.

Liao, T. (2015). Augmented or admented reality? The influence of marketing on augmented reality technologies. Information Communication \& Society, 18(3), 310-326.

Nielsen (2013). THE MOBILE CONSUMER A GLOBAL SNAPSHOT. http://www.nielsen.com/content/dam/corporate/us/en/ reports downloads/2013\%20Reports/MobileConsumer-Report-2013.pdf

Sevillano-Garcia, M.L y Vázquez -Cano. E. ( 2015) Modelos de investigación en contextos ubicuos y móviles en Educación Superior.. Madrid. McGrawHill

UNESCO (2013). UNESCO Policy Guidelines for Mobile Learning. Paris: United Nations Educational, Scientific and Cultural Organization

Webcertain (2014). The Webcertain Global Search and Social Report 2014. http://internationaldigitalhub.com/en/publications/the -webcertain-global-search-and-social-report-2014 